\title{
EVALUATION OF URINE N1,N12-DIACETYLSPERMINE AS POTENTIAL TUMOR MARKER FOR URINARY BLADDER CANCER
}

\author{
David Stejskal ${ }^{\mathrm{a}}$, Viera Humenanska ${ }^{\mathrm{b}}$, Zuzana Hanulova $^{\mathrm{b}}$, Richard Fiala ${ }^{\mathrm{c}}$, Radovan Vrtal ${ }^{\mathrm{c}}$, \\ Pavlina Solichova ${ }^{\mathrm{a}}$, Michal Karpisek ${ }^{\mathrm{d}}$
}

\author{
a Department of Laboratory Medicine, Sternberk Hospital, Czech Republic \\ b Gnosis s.r.o., Bratislava, Slovakia \\ c Department of Urology, Faculty Hospital Olomouc \\ $d$ University of Veterinary and Pharmaceutical Sciences, Brno \\ e-mail: stejskal@nemstbk.cz
}

Received: September 3, 2006; Accepted (with revision) November 29, 2006

Key words: Urine N1,N12-Diacetylspermine/Urinary Bladder Cancer/ELISA

Background: N1,N12-diacetylspermine, a diacetylpolyamine which was recently identified in urine, appeared to be a useful tumor marker for a number of cancers. No valid data on urine diacetylspermine concentration in patients with urinary bladder cancer exist.

Aim: Evaluation of urine N1,N12-diacetylspermine concentrations in individuals with urinary bladder cancer.

Methods: Urine samples were used from 36 patients with urothelial tumors of the urinary bladder and from 30 patients with benign urological diseases. Urine was collected before cystoscopy. Enzyme-linked immunoabsorbent assays (ELISA) were performed for diacetylspermine from urine.

Results: Urine diacetylspermine did not differentiate in individuals with urinary bladder cancer from controls (medians 171.5 vs $143.8, \mathrm{p}=0.64$ ). Its efficacy for urinary bladder cancer detection was not shown.

Conclusions: Urine $N 1, N 12$-diacetylspermine is probably not a useful marker for urinary bladder cancer.

\section{INTRODUCTION}

Urothelial urinary bladder cancer is a disease with a variable clinical course ${ }^{1}$. Cystoscopy and cytology are standard methods used in diagnosis and follow-up of tumors of the urinary bladder. Cytology requires correct sample processing and is a highly subjective interpretation done by a cytopathologist. Results are available minimally after 24 hours and display a low specificity (40-60\%). Cystoscopy is uncomfortable, the hence other methods are being tested.

To date, several papers have been published on examination using several laboratory markers (e.g. measurement of TPS, BTA, telomerase, NMP22, M344, chromosomes aneuploidy, survivin, livin, midkine, endostatin). Although the first studies reported on decreased frequency of cystoscopic examinations and satisfactory diagnostic sensitivity and specificity of these markers, further investigations failed to confirm the promising conclusions. The markers were found only to supplement cystoscopic examination, not replace it $^{1-13}$.

In addition, interpretation of any laboratory marker in clinical practice comprises a false positivity of results. This may be associated with the presence of tumor cells in urine released from a tumor, which is as yet endoscopically occult. There is sporadic information about the comparison of the course of disease in positive and false-positive patients ${ }^{3}$. Therefore, additional markers are being sought after.
The aim of our study was to test the diagnostic efficacy of a new potential hypothetic cancer marker - diacetylspermine concentrations in the urine individuals with urinary bladder cancer ${ }^{14}$.

\section{MATERIAL AND METHODS}

The project was designed as a prospective blind study. 69 patients of the Urology Clinic Department at the Olomouc Faculty Hospital were evaluated. 66 subjects were selected (mean age 63.1 years). They were divided into 2 groups:

$1^{\text {st }}$ group - 36 patients with endoscopic and histologic urinary cancer bladder confirmation

$2^{\text {rd }}$ group - 30 patients with benign urologic disorders and negative cancer anamnesis.

\section{Sampling}

Urine was sampled before cystoscopy and tested for urine microscopy, cytology and frozen for diacetylspermine measurement.

\section{ELISA for diacetylspermine}

The human ELISA kits were obtained from Biovendor Laboratory Medicine, Inc. (Brno, Czech Republic). The assay was conducted according to the manufacturer's instructions. The intra-assay and inter-assay variations were 
Table 1. Urine N1,N12-diacetylspermine concentrations in subgroups by urine bladder cancer diagnosis.

\begin{tabular}{|l|c|c|c|c|c|c|}
\hline Parametr & $\mathrm{X}$ & Median & SD & Normality & RR & $\mathrm{n}$ \\
\hline DAC-A & 303.1 & 171.5 & 391.8 & No & A-B; $\mathrm{P}=0.52 ; \mathrm{Z}=0.64$ & 36 \\
\hline DAC-B & 353.3 & 143.8 & 743.3 & No & & 30 \\
\hline
\end{tabular}

DAC-A: diacetylspermine in individuals with bladder cancer (immunohistochemistry)

DAC-B: diacetylspermine in individuals without bladder tumor (with benign urological disease)

RR: $\quad$ significant diference Mann-Whithey test for independent samples was used to test differences between groups.

Table 2. $\chi^{2}$ in frequency table; patients divided by urine diacetylspermine concentration (Subgroups: $1=<80,2=80-210,3=210-340,4=>340 \mathrm{nmol} / 1$ ).

\begin{tabular}{|l|c|c|c|c|c|}
\hline & $<80(1)$ & $80-210(2)$ & $210-340(3)$ & $>340(4)$ & Sum (\%) \\
\hline Cancer bladder yes & 8 & 12 & 6 & 10 & $36(54.5 \%)$ \\
\hline Cancer bladder no & 7 & 12 & 3 & 8 & $30(45.5 \%)$ \\
\hline Sum $(\%)$ & $15(22.7 \%)$ & $24(36.4 \%)$ & $9(13.6 \%)$ & $18(27.3 \%)$ & $66(100 \%)$ \\
\hline
\end{tabular}

$\chi^{2} 0.13, p=0.71$

evaluated by measuring 3 different samples in 10 replicates (CV intra-assay $<5 \%$, CV inter-assay $<7 \%$, detection limit $3 \mathrm{nmol} / \mathrm{l})$.

\section{Statistical analysis}

The data were processed by means of the software Medcalc (Medcalc, Mariakerke, Belgium). $\mathrm{P}<0.05$ was considered as statistically significant. The comparison of cancer marker values between subjects with and without cancer diagnosis was made using a Mann-Whitney test (for none normal data distribution) and independent $\mathrm{t}$ test (normal data distribution). ROC curves for normal data distribution and frequency table with chi-square for none normal data distribution were performed. All the data are expressed as medians and means \pm S.D.

\section{RESULTS}

Urine values of diacetylspermine did not differ in individuals with urinary bladder cancer and controls (medians 171.5 vs 143.8, $\mathrm{p}=0.64)$, efficacy was not sufficient (AUC < 0.7) (Tab. 1-2, Fig. 1).

\section{DISCUSSION}

Cell growth is dependent on a sustained supply of polyamines, which is typically met by the integrated contributions of biosynthesis, catabolism, uptake, and export, each of which is sensitively regulated by effector molecules that, in turn, are controlled by intracellular polyamine pools. Thus, ornithine decarboxylase and $S$-adenosylmethionine decarboxylase control biosynthesis, a polyamine transport system modulates uptake, and spermidine/sper-

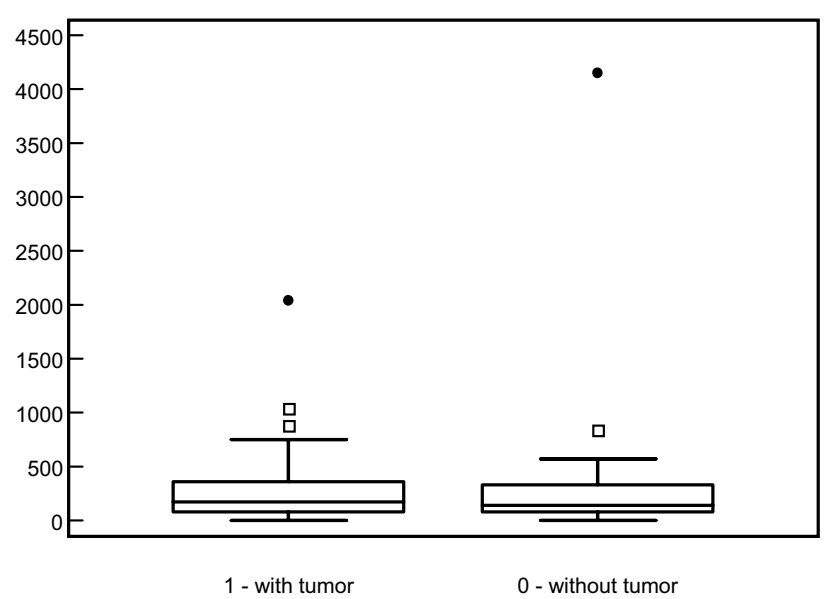

Fig. 1. Urine $N 1, N 12$-diacetylspermine concentrations in subgroups by urinary bladder cancer diagnosis $(\mathrm{y}$ axis $=$ diacetylspermine concentrations in $\mathrm{nmol} / 1)$.

mine $N^{1}$-acetyltransferase regulates polyamine catabolism and export from the cell. Neoplastic cell growth is associated with elevated polyamine biosynthetic activity, even when the surrounding normal tissue itself is rapidly proliferating $^{15-17}$.

$N 1, N 12$-diacetylspermine (DiAcSpm) is a minor component of human urinary polyamine to which little attention has been paid until recently. Excretion of this diacetylpolyamine, in particular, into urine is frequently and markedly increased in association with all types of cancer (prostate, breast, colon, etc.). Remission is usually accompanied by recovery of urinary diacetylpolyamine to normal level. Diacetylspermine is more sensitive than CEA, Ca 19-9 and $\mathrm{Ca}$ 15-3 for detecting colorectal and breast cancer ${ }^{13,14}$. More importantly, diacetylspermine ef- 
ficiently detects cancer at early stages, is highly sensitive for cancer and may serve as a prognostic indicator and marker for recurrence of prostate, urogenital, liver, breast, stomach and colon cancers ${ }^{14-18}$.

Thus, to the author's knowledge, it is unclear whether diacetylspermine is also involved and the possible roles of diacetylspermine urine concentration in the diagnosis of the urinary bladder cancer. No information about diacetylspermine role in urinary bladder cancer expansion was found in the literature.

An ELISA procedure for rapid determination of diacetylspermine was recently developed to promote the clinical application of this new tumor marker ${ }^{19}$. Results of our paper did not confirm the hypothesis about this marker use in clinical urinary bladder cancer diagnosis.

It is concluded that individuals with urinary bladder cancer did not differ in urine $N 1, N 12$-diacetylspermine values from healthy controls. Our data suggest, for the first, that urine diacetylspermine is not the sufficient marker for bladder cancer diagnosis.

\section{ACKNOWLEDGEMENT}

This study was supported by IGA grants NR/8402 a NR/ 8442 from the Ministry of Health of the Czech Republic.

\section{REFERENCES}

1. Ozen H, Hall MC. Bladder cancer. Curr Opin Oncol 2000; 12:2559.

2. Sharp JD, Hausladen DA, Maher MG, Wheeler MA, Altieri DC, Weiss RM. Bladder cancer detection with urinary survivin, an inhibitor of apoptosis. Front. Biosci 2002; 7:36-1.

3. Stein JP, Grossfeld GD, Ginsberg DA. Prognostic markers in bladder cancer: a contemporary review of the literature. J Urol 1998; 160:645-59.

4. Boman H, Hedelin H, Holmang S. Four bladder tumor markers have a dissappointing senzitivity for small size and low grade reccurence. J Urol 2002; 167:80-3.
5. Pode D, Golijanin D, Sherman Y. Immunostaining of Lewis $X$ in cells from voided urine. J.Urol 1998; 159:389-2.

6. Sarosdy MF, de Vere White RV, Soloway MS. Results of multicentric trial using the BTA test to monitor for and diagnose reccurent bladder cancer. J.Urol 1995; 154:379-3.

7. Soloway MA, Briggman JV, Carpinito GA. Use of a new tumor marker, urinary NMP22, in the detection of occult or rapidly recurrent transitional cell carcinoma of the urinary tract following surgical treatment. J Urol 1996; 156:363-7.

8. Friedrich MG, Helstern A, Toma MI. Are false-positive urine markers for the detection of bladder carcinoma really wrong or do they predict tumor recurrence? EurUrol 2003; 43:146-1.

9. Mirkin BL, Clark S, Zheng X, Chu F. Identification of midkine as a mediator for intercellular transfer of drug resistance. Oncogene $2005 ; 24: 4965-4$

10. Munz M, Zeidler R, Aires O. The tumor-associated antigen EpCAM upregulates the fatty acid binding protein E-FABP. Canc Let 2005; 225:151-7.

11. Schmidt A, Sommer F, Reiner M. Differential endostatin binding to bladder, prostate and kidney tumour vessels. BJU Int 2005; 95:174-9.

12. Du Z, Hou S. The anti-angiogenic activity of human endostatin inhibits bladder cancer growth and its mechanism. J Urol 2003; 170:2000-3.

13. Kawakita M, Hiramatsu K. Diacetylated derivatives of spermine and spermidine as novel promising tumor markers. J Biochem 2006; 139:315-2.

14. Kawakita M, Hiramatsu K, Sugimoto M. Clinical usefulness of urinary diacetylpolyamines as novel tumor markers. Rinsho Byori 2004; 52:321-7.

15. Enjoji M, Nakamuta $M$, Arimura E. Clinical significance of urinary $\mathrm{N} 1, \mathrm{~N} 12$-diacetylspermine levels in patients with hepatocellular carcinoma. Int J Biol Markers 2004; 19:322-7.

16. Sugimoto M, Hiramatsu K, Kamei S. Significance of urinary N1,N8diacetylspermidine and N1,N12-diacetylspermine as indicators of neoplastic diseases. J Cancer Res Clin Oncol 1995; 121:317-9.

17. Kubota S, Yamasaki Z, Yoshimoto M.The value of urinary polyamine assay in stomach cancer. Comparison with serum carcinoembryonic antigen. Cancer 1985; 56:1630-1635.

18. Kee K, Vujcic S, Merali S. Metabolic and antiproliferative consequences of activated polyamine catabolism in $\mathrm{LNCaP}$ prostate carcinoma cells. J Biol Chem 2004; 279:27050-8.

19. In: http://www.biovendor.com 